\title{
Advanced gastric carcinoma successfully treated with TS-1 as neoadjuvant chemotherapy
}

\author{
Takaki Yoshikawa ${ }^{1}$, Masahiro Kanari ${ }^{1}$, Akira Tsuburaya ${ }^{1}$, Osamu Kobayashi ${ }^{1}$, Motonori Sairenji ${ }^{1}$, \\ Hisahiko Motohashi ${ }^{1}$, Yoichi Kameda ${ }^{2}$, and Yoshikazu Noguchi ${ }^{3}$ \\ ${ }^{1}$ Department of Gastrointestinal Surgery, Kanagawa Cancer Center, 1-1-2 Nakao, Asahi-ku, Yokohama 241-0815, Japan \\ ${ }^{2}$ Department of Pathology, Kanagawa Cancer Center, Yokohama, Japan \\ ${ }^{3}$ Department of Surgery, Yokohama Kowan Hospital, Yokohama, Japan
}

\begin{abstract}
We report a patient with advanced gastric carcinoma successfully treated with the novel oral fluoropyrimidine anticancer drug TS-1 as neoadjuvant chemotherapy. The patient was a 76-year-old man who had gastric cancer clinically diagnosed as N2T4, invading the pancreas, the duodenum, and the transverse colon. He was treated as an outpatient with TS-1, $120 \mathrm{mg}$, administered orally every day for 28 days, followed by 14 days' rest, as one course. Two courses resulted in a marked reduction of the tumor without severe toxicity. Subsequently, the patient underwent curative surgery consisting of distal gastrectomy with D2 lymph node dissection. No surgical complications were observed. On microscopic examination, a few tumor cells were detected in the ulcer scar of the resected stomach and in the regional lymph nodes. Our report is the first to demonstrate the advantages of TS-1 as neoadjuvant chemotherapy for the treatment of advanced gastric carcinoma.
\end{abstract}

Key words Gastric carcinoma · TS- 1 - Neoadjuvant chemotherapy

\section{Introduction}

The prognosis of patients with unresectable gastric cancer is poor. To prolong their survival, chemotherapy followed by curative resection has been proposed [1]. To date, intensive intravenous chemotherapy has been employed as the neoadjuvant chemotherapy [2-7]. However, some patients cannot undergo surgery because of severe toxicity of the chemotherapy or because of tumor progression [2-7]. A high response rate with

Offprint requests to: $\mathrm{T}$. Yoshikawa

Received: July 6, 2000 / Accepted: October 4, 2000 an acceptable toxicity is required in the setting of neoadjuvant chemotherapy. Since the introduction of the novel oral fluoropyrimidine anticancer drug TS-1, it has been tested in our department as one of the candidates for first-line chemotherapy for unresectable and recurrent gastric cancer, because it was the only oral agent with a high response rate and acceptable toxicities. Here we report a patient with advanced gastric carcinoma who was successfully treated with TS-1 as neoadjuvant chemotherapy.

\section{Case report}

A 76-year-old man with a 3-month history of epigastric discomfort was referred to the Kanagawa Cancer Center Hospital. On physical examination, a tumor was palpable in the upper abdomen. Endoscopy and upper gastrointestinal tract series demonstrated type 3 advanced gastric carcinoma invading the antrum and the duodenum (Fig. 1A,B). A biopsy specimen showed moderately differentiated tubular adenocarcinoma. An abdominal computed tomography (CT) scan showed that the gastric tumor had invaded the duodenum, pancreas, and regional lymph nodes (Fig. 2A). To further assess the extent of tumor spread, laparoscopy was performed. Laparoscopy showed that the gastric tumor had invaded the duodenum and transverse colon. No peritoneal dissemination was observed. Peritoneal lavage cytology revealed that there were no tumor cells in the abdominal cavity. The patient was diagnosed with advanced disease ( $\mathrm{cP} 0, \mathrm{cH} 0, \mathrm{cT} 4, \mathrm{cN} 2$; cStage IV according to the Japanese classification of gastric carcinoma, 2nd English edition [8]).

TS-1, $120 \mathrm{mg}$, was administered orally every day for 28 days, followed by 14 days' rest, as one course. Two courses resulted in a marked reduction of the tumor. Only grade 1 anemia (according to the WHO toxicity criteria) was observed during the chemotherapy. All the 
A
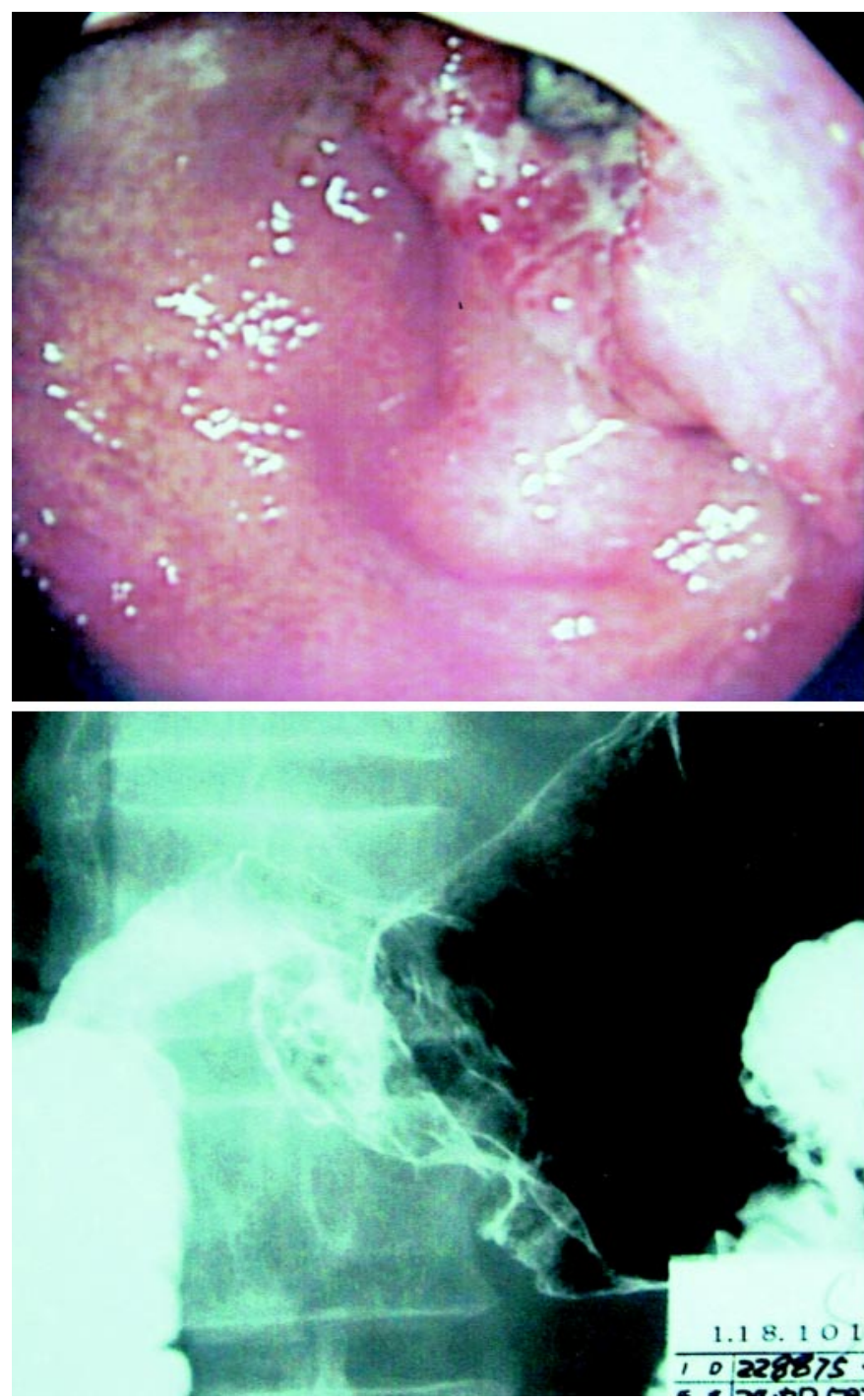

Fig. 1A-D. Endoscopy (A, C) and upper gastrointestinal tract series (B, D) demonstrated type 3 advanced gastric carcinoma invading the duodenum before treatment $(\mathbf{A}, \mathbf{B})$.
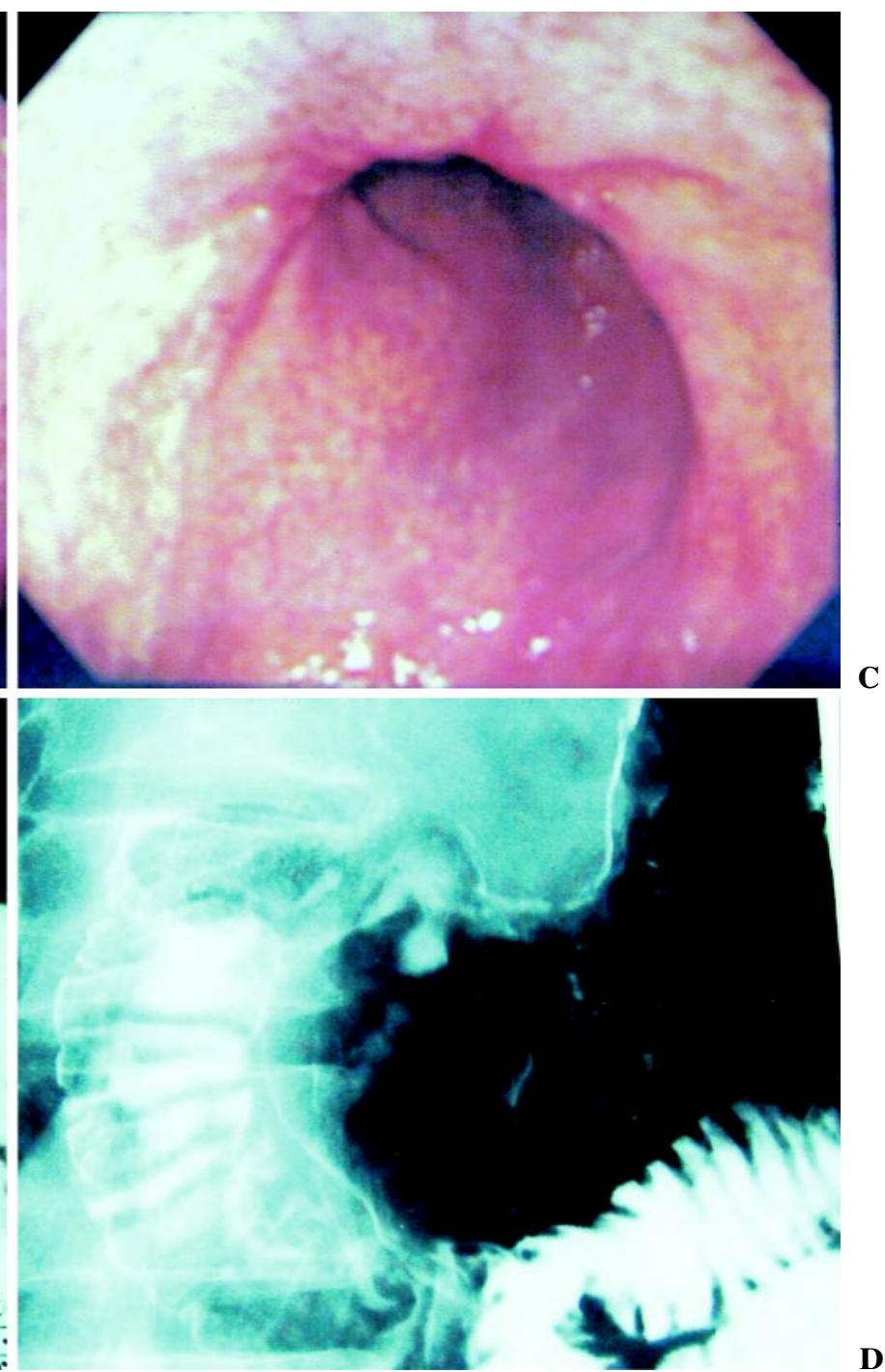

After treatment (C, D) only the gastric ulcer scar was demonstrated treatment was performed at the outpatient clinic. Endoscopy and upper gastrointestinal tract series revealed gastric ulcer scars (Fig. 1C,D). On the abdominal CT scan after treatment, the invasion to the duodenum had become unclear, and the regional lymph node had decreased in size (Fig. 2B).

Three weeks after the completion of the chemotherapy, the patient was operated on. Soft induration due to fibrosis was palpable on part of the bulbar wall of the duodenum. Neither invasion nor adhesion was observed between the primary tumor and the pancreas or the transverse colon. Peritoneal lavage cytology demonstrated no cancer cells in the abdominal cavity. The patient underwent curative resection, consisting of distal gastrectomy at a normal bulbus with D2 lymph node dissection, without the resection of any other organ. Macroscopically, ulcer scars were observed in the antrum (Fig. 3). On microscopic examination, a few tumor cells were detected in the ulcer scar of the resected stomach and in the regional lymph nodes (Fig. 4). There were no tumor cells on the surgical margins of the resected specimens. Final findings showed that the tumor was stage IIIb, P0, H0, T3, N2 [8]. No postsurgical complications were observed.

Four months after surgery, the patient is alive without recurrence. He is receiving adjuvant chemotherapy with TS-1, as an outpatient, and has developed no adverse reactions. 
$\mathbf{A}$

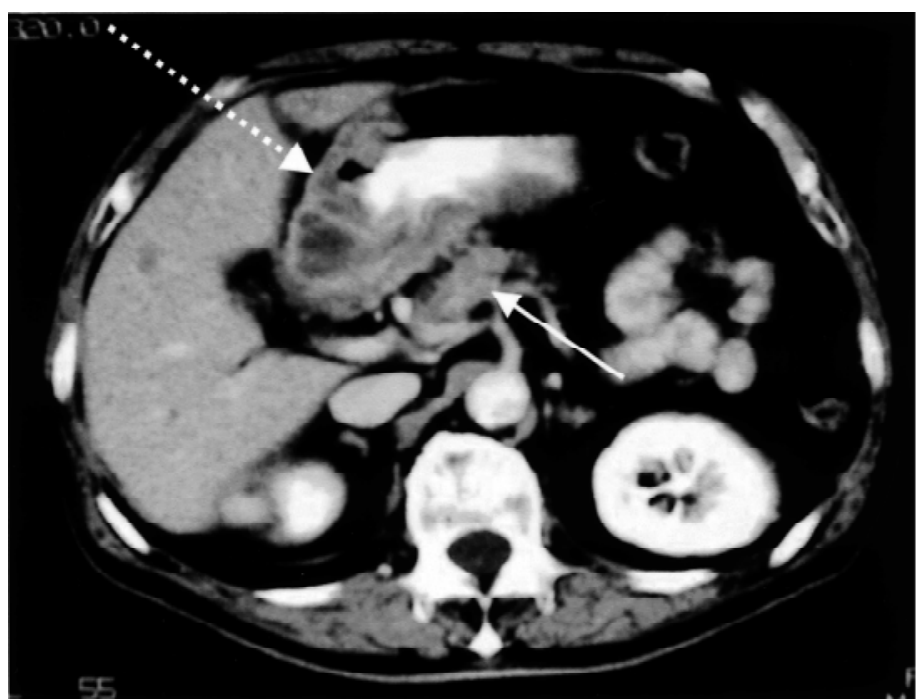

Fig. 2A,B. Abdominal computed tomography (CT) scan demonstrating gastric tumor invading the duodenum (broken line arrows), pancreas, and regional lymph nodes (continuous

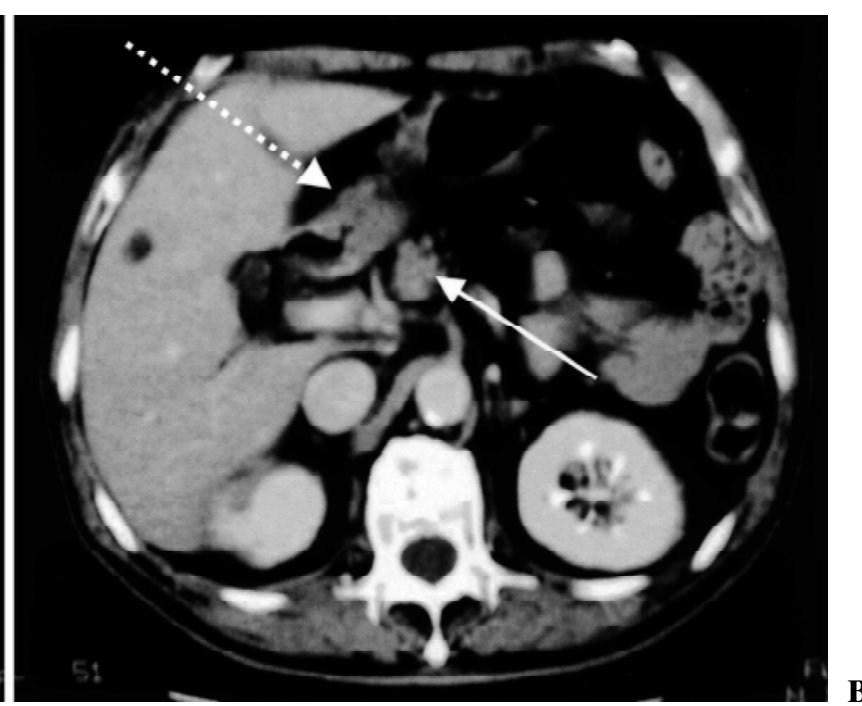

line arrows) before $(\mathbf{A})$ and after $(\mathbf{B})$ treatment. The invasion to the duodenum had become unclear and the size of the regional lymph node was reduced after the treatment $(\mathbf{B})$

\section{Discussion}

From the preoperative evaluations we diagnosed that the tumor was cStage IV, cP0, cH0, cT4, cN2. The prognosis of patients at stage IV was reported to be extremely poor [9]. Even after resection for cure, there were only a few survivors. To overcome this difficult problem, several investigators have employed pancreaticoduodenectomy for advanced gastric cancer invading the pancreas or duodenum, and some therapeutic effects have been reported [10-12]. However, there were few survival benefits for those patients with tumors with distant lymph node metastases, because such tumors had already spread to other distant organs or to the peritoneum [10-12]. Thus, extended surgery alone is not sufficient, and a new strategy has to be considered for these tumors. One possible approach may be neoadjuvant chemotherapy. As a pilot study, we treated this patient with TS-1 preoperatively.

Our patient showed surprising reduction of the tumor, without severe toxicity, after chemotherapy with TS-1. TS-1 is a novel oral anticancer drug, composed of tegafur (FT), gimestat (CDHP), and otastat potassium (Oxo) in a molar ratio of 1:0.4:1, based on the biochemical modulation of 5-fluorouracil (5-FU) [13]. CDHP inhibits dihydropyrimidine dehydrogenase (DPD), an enzyme which degrades 5-FU, and maintains prolonged 5-FU concentrations in the blood and tumors [14]. Oxo is distributed in the gastrointestinal tract at a high concentration after oral administration, and alleviates the gastrointestinal toxicity of 5-FU [15]. TS-1 improves the tumor-selective toxicity of 5-FU, by the actions of the two modulators, CDHP and Oxo. The response rate of TS-1 was reported to be $54 \%$ in an early phase II study [16] and $49 \%$ and $44 \%$ in late phase II studies [17,18]. In these reports, major adverse reactions were gastrointestinal symptoms and myelosuppression, with little severe toxicity [16-18]. The results suggested that TS-1 was suitable for outpatients because of its mild toxicity and high response rate $[17,18]$.

In a setting of neoadjuvant chemotherapy, the regimen used should not induce severe toxicity that may delay surgery, while it should induce a high response rate, to improve the resectability of the tumor. To date, several intensive intravenous chemotherapeutic regimens, including EAP, FLP, FLEP, and PMUE, have been used as neoadjuvant chemotherapy [2-7]. Intensive chemotherapy usually requires hospital care, because of its serious side effects, resulting in an impaired quality of life. It was reported that some patients who received neoadjuvant chemotherapy could not undergo surgical resection because of severe toxicity or tumor progression [2-7]. Until TS-1 was developed, there was no oral agent with a high response rate and acceptable toxicities.

As demonstrated in this case report, our patient was treated with TS-1, as an outpatient, and TS-1 induced dramatic reduction of the tumor, without serious toxicity. Moreover, operation was not delayed and no surgical complications were observed. Our report is the first to demonstrate the advantages of TS- 1 as neoadjuvant chemotherapy for the treatment of advanced gastric carcinoma. 

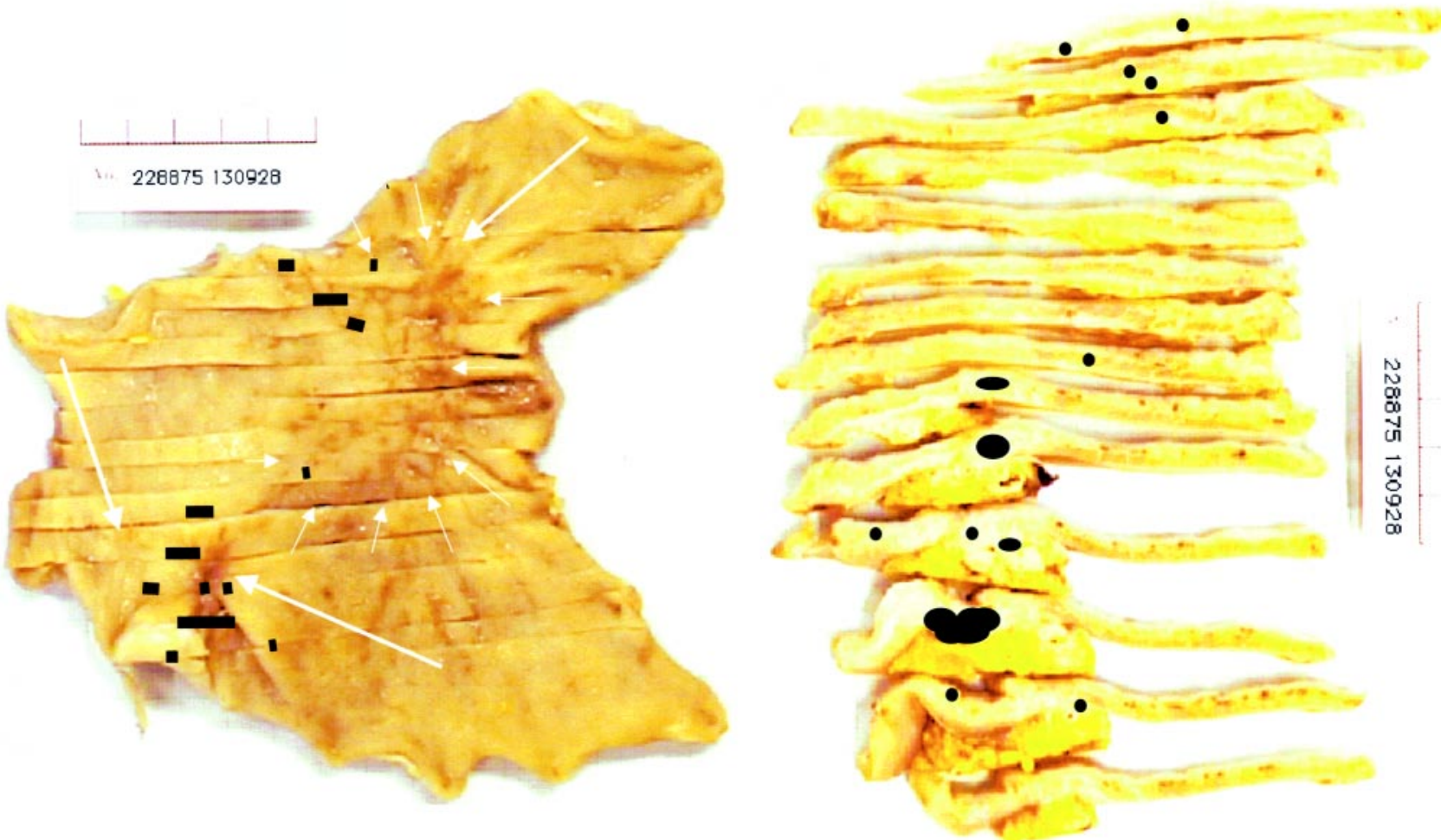

$\mathbf{A}$

Fig. 3A,B. Photographs of resected stomach, showing ulcer scars (white arrows) on horizontal section (A) and vertical section (B); black areas indicate lesions occupied by residual tumor cells, detected by microscopy

$\mathbf{A}$
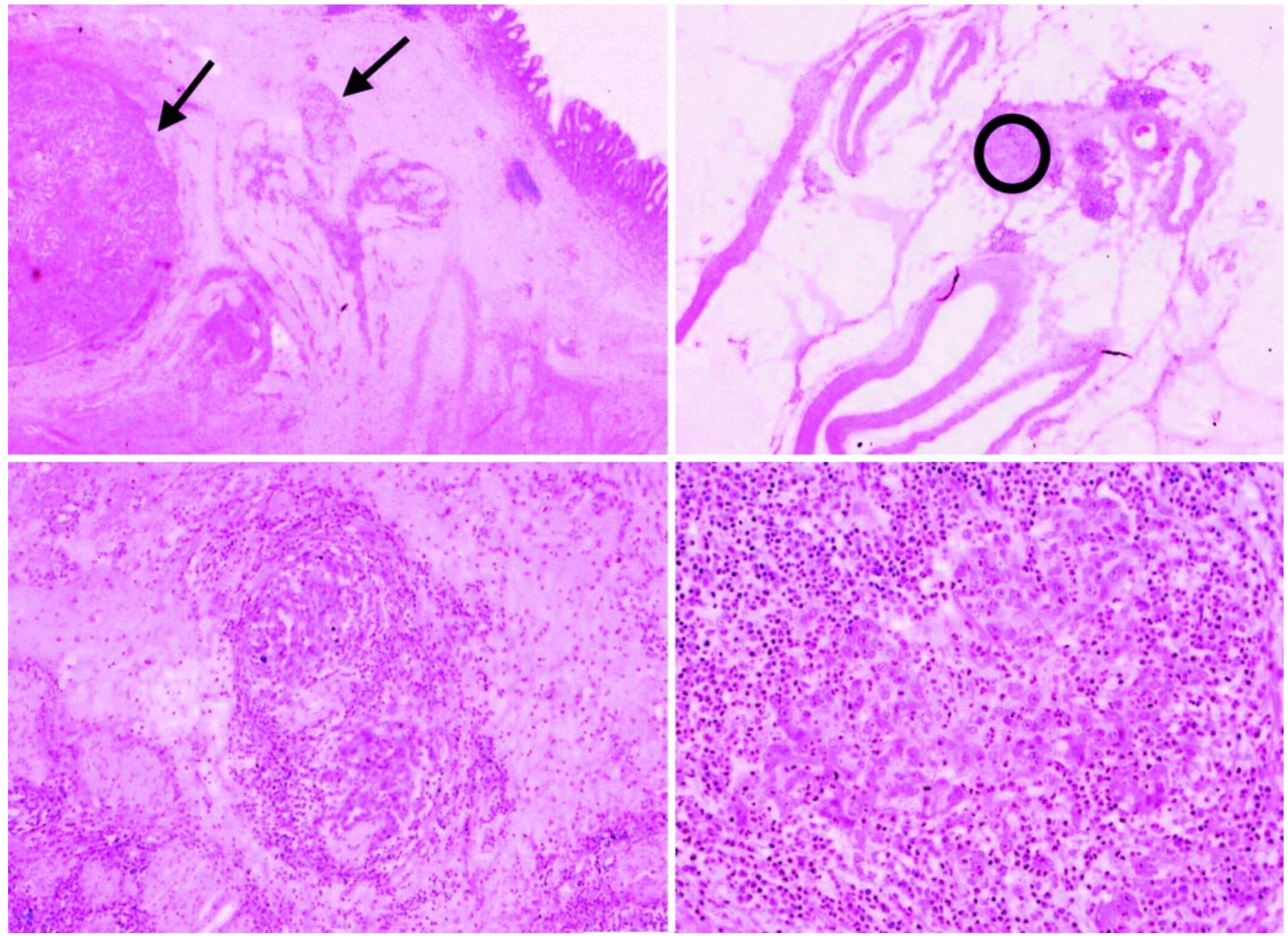

Fig. 4A-D. Photomicrographs of resected stomach, showing the residual tumor cells in the granulation tissue. $\mathbf{B}$ is a magnification of the lesion indicated by black arrow on the right in A. C and D Photomicrographs of the dissected lymph node that was reduced in size after the treatment; $\mathbf{D}$ is a magnification of the lesion indicated by the black circle in $\mathbf{C} ; \mathbf{D}$ shows residual tumor cells in the lymph node surrounded by loose connective tissue. 


\section{References}

1. Frei E, Miller D, Clark JR, Fallon BG, Ervin TJ. Clinical and scientific considerations in preoperative (neoadjuvant) chemotherapy. Recent Results Cancer Res 1986;103:1-5.

2. Wilke H, Preusser P, Fink U, Gunzer U, Meyer HJ, Meyer J, et al. Preoperative chemotherapy in locally advanced and nonresectable gastric cancer: a phase II study with etoposide, doxorubicin, and cisplatin. J Clin Oncol 1989;7:1318-26.

3. Plukker JT, Mulder NH, Sleijfer DT, Grond J, Verschueren RC. Chemotherapy and surgery for locally advanced cancer of the cardia and fundus: phase II study with methotrexate and 5fluorouracil. Br J Surg 1991;78:955-8.

4. Rougier P, Mahjoubi M, Lasser P, Ducreux M, Oliveria J, Ychou $\mathrm{M}$, et al. Neoadjuvant chemotherapy in locally advanced gastric carcinoma - a phase II trial with combined continuous intravenous 5-fluorouracil and bolus cisplatinum. Eur J Cancer 1994; 30A:1269-75.

5. Fink U, Schuhmacher C, Stein HJ, Busch R, Feussner H, Dittler HJ, et al. Preoperative chemotherapy for stage III-IV gastric carcinoma: feasibility, response and outcome after complete resection. Br J Surg 1995;82:1248-52.

6. Yonemura Y, Sawa T, Kinoshita K, Matsuki N, Fushida S, Tanaka S, et al. Neoadjuvant chemotherapy for high-grade advanced gastric cancer. World J Surg 1993;17:256-61.

7. Nakajima T, Ota K, Ishihara S, Oyama S, Nishi M, Ohashi Y, et al. Combined intensive chemotherapy and radical surgery for incurable gastric cancer. Ann Surg Oncol 1997;4:203-8.

8. Japanese Gastric Cancer Association. Japanese classification of gastric carcinoma. 2nd English ed. Gastric Cancer 1998;1:10-24.

9. Aiko T, Sasako M. The new Japanese classification of gastric carcinoma: points to be revised. Gastric Cancer 1998;1:25-30.

10. Nomoto K, Nashimoto K, Tsuchiya Y, Makino H, Tsutsui M, Tanaka O, et al. Significance of pancreaticoduodenectomy for advanced gastric cancer with pancreatic invasion (in Japanese). Jpn J Gastroenterol Surg 1998;59:908-13.

11. Ohashi I, Takahashi T, Ota H, Takagi K, Nishi M, Kajitani T. Combined resection of adjacent organs for advanced cancer of the stomach. Pancreaticoduodenectomy and left upper abdominal evisceration (in Japanese). Surgical Therapy 1985;52:17380 .

12. Nishi M, Nakajima T. Pancreaticoduodenectomy for the treatment of gastric cancer (in Japanese). Surgery 1970;32:887-94.

13. Shirasaka T, Nakano K, Fukushima M, Satake H, Uchida J, Fujioka A, et al. Antitumor activity of $1 \mathrm{M}$ tegafur $-0.4 \mathrm{M} 5-$ chloro -2, 4-dihydroxypyrimidine $-1 \mathrm{M}$ potassium oxonate (S-1) against human colon carcinoma orthotopically implanted into nude rats. Cancer Res 1996;56:2602-6.

14. Shirasaka T, Shimamoto Y, Fukushima M, et al. Development of a novel form of an oral 5-flurorurail delivative (S-1) directed to the potentiation of the tumor-selective cytotoxicity of 5fluorouracil by two biochemical modulators. Anticancer Drugs 1996;7:578-57.

15. Takechi T, Nakano K, Uchida J, Mita A, Toko K, Takeda S, et al. Antitumor activity and low intestinal toxicity of S-1, a new formulation of oral tegafur, in experimental tumor models in rats. Cancer Chemother Pharmacol 1997;39:205-11.

16. Horikoshi N, Mitachi Y, Sakata Y, et al. S-1, a new oral fluoropyrimidine is very active in patients with advanced gastric cancer (early phase II study) (abstract). Proc Am Soc Clin Oncol 1996;15:466.

17. Sakata Y, Ohtsu A, Horikoshi N, Sugimachi K, Mitachi Y, Taguchi T. Late phase II study of novel oral fluoropyrimidine anticancer drug S-1 (1M tegafur $-0.4 \mathrm{M}$ gimestat $-1 \mathrm{M}$ otastat potassium) in advanced gastric cancer patients. Eur J Cancer 1998;34:1715-20.

18. Koizumi W, Kurihara M, Nakano S, Hasegawa K. Phase II study of S-1, a novel oral derivative of 5-fluorouracil, in advanced gastric cancer. Oncology 2000;58:191-7. 